\title{
Generic MS-based method for the bioanalysis of therapeutic monoclonal antibodies in nonclinical studies
}

\author{
Noritaka Hashii*,1, Yoshiko Tousaka1, Koji Arai², Ryoya Goda³, Noriko Inoue ${ }^{4,8}$, Kazuyuki \\ Murata $^{5}$, Takeshi Okuzono ${ }^{6}$, Satomi Sasahara ${ }^{4}$, Takuma Shigeyama $^{5}$, Hidehisa Tachiki ${ }^{4}$, \\ Shinichi Yamane ${ }^{6}$, Yoshiro Saito ${ }^{7}$ \& Akiko Ishii-Watabe ${ }^{1}$ \\ ${ }^{1}$ Division of Biological Chemistry and Biologicals, National Institute of Health Sciences, 3-25-26 Tonomachi, Kawasaki-ku, \\ Kawasaki, Kanagawa 210-9501, Japan \\ 2Bioanalysis Department, Advanced Technology Center, Medical Solution Segment, LSI Medience Corp., 30-1, Shimura 3-chome, \\ Itabashi-ku, Tokyo 174-8555, Japan \\ ${ }^{3}$ Drug Metabolism \& Pharmacokinetics Research Laboratories, R\&D Division, Daiichi Sankyo Co., Ltd, 1-2-58 Hiromachi, \\ Shinagawa-ku, Tokyo 140-8710, Japan \\ ${ }^{4}$ Scientific Research and Business Development Department, Towa Pharmaceutical Co., Ltd, Kyoto Research Park KISTIC \#202, 134 \\ Chudoji Minami-machi, Shimogyo-ku, Kyoto 600-8818, Japan \\ ${ }^{5}$ Osaka Laboratory, Technical Solution Headquarters Sumika Chemical Analysis Service, Ltd, 1-135, Kasugade-naka 3-chome, \\ Konohana-ku, Osaka 554-0022, Japan \\ ${ }^{6}$ Drug Development Solutions Center, Sekisui Medical Co., Ltd, 2117 Muramatsu, Tokai-mura, Naka-gun, Ibaraki 319-1182, Japan \\ ${ }^{7}$ Division of Medical Safety Science, National Institute of Health Sciences, 3-25-26 Tonomachi, Kawasaki-ku, Kawasaki, Kanagawa \\ 210-9501, Japan \\ ${ }^{8}$ Current address: Scientific Research and Business Development Department, Towa Pharmaceutical Co., Ltd, National Cerebral \& \\ Cardiovascular Center \#30602, 6-1, Kishibe Shinmachi, Suita, Osaka 564-8565, Japan \\ *Author for correspondence: Tel.: +81 44270 6516; hashii@nihs.go.jp
}

\begin{abstract}
Aim: A generic bioanalytical method was developed to quantify therapeutic IgG1 monoclonal antibodies (mAbs) in mouse sera by combining an easy sample preparation method with LC/MS using selected reaction monitoring. Materials \& methods: Rituximab and trastuzumab were used as model mAbs. A synthetic stable isotope-labeled peptide or a stable isotope-labeled mAb was used as an internal standard. The method feasibility was evaluated by a collaborative study involving six laboratories. Results: The calibration curve ranged from 1.0 to $1000.0 \mu \mathrm{g} / \mathrm{ml}$ (correlation coefficient >0.99). The validation parameters including selectivity, linearity of calibration curve, accuracy and precision met the predefined acceptance criteria. Conclusion: Our method is a useful bioanalytical method for the quantification of therapeutic IgG mAbs in nonclinical animal studies.
\end{abstract}

First draft submitted: 8 October 2019; Accepted for publication: 30 January 2020; Published online: 24 February 2020

Keywords: $\mathrm{LC} / \mathrm{MS} \bullet$ monoclonal antibody $\bullet$ mouse serum $\bullet$ selected reaction monitoring

The therapeutic monoclonal antibody (mAb) is one of the most successful biological therapeutics [1]. As of 2019, $>50 \mathrm{mAbs}$ were approved in the USA, the EU and Japan $[2,3]$. The rapid growth of therapeutic $\mathrm{mAb}$ applications has increased demand for the bioanalysis of $\mathrm{mAb}$ therapeutics. A common bioanalysis technique for $\mathrm{mAbs}$ is the ligand-binding assay (LBA), which includes ELISA $[4,5]$. LBAs are very sensitive and able to measure protein levels as low as the picogram/milliliter level. However, suitable capture reagents for LBAs needed to be developed, which takes time and resources, and the reagents may not be obtained at an early stage of drug development. In addition, measurements by LBAs may be affected by the presence of antidrug antibodies in matrices [6].

Recently, LC/MS has become an important alternative method to LBA for biological therapeutics [7]. The most popular bioanalytical MS method is LC-MS/MS using SRM mode (LC/MS/MS-SRM) on a triple quadrupole mass spectrometer. The LC/MS/MS-SRM assay is already widely used for the quantitative bioanalysis of smallmolecule drugs, and has the advantages of high selectivity, a wide dynamic range, and short method development time. In general, the bioanalysis of therapeutic mAbs by LC/MS/MS-SRM is performed as follows: the target 
$\mathrm{mAb}$ is purified from blood samples and digested to peptides, and the resulting signature surrogate peptide(s) is quantified by LC/MS/MS-SRM [8,9]. The surrogate peptide sequence must be unique to the analytical target proteins in matrices, for example, tryptic peptides from the complementarity determining regions (CDR-peptides) are often used as mAb surrogates. An LC/MS/MS-SRM format using a CDR-peptide could be a useful quantitative bioanalytical method for both nonclinical and clinical studies, and would therefore have a shortened development period. However, this approach could be challenging for the development of a sample preparation method because of the multiple/complicated processes involving affinity purification. In addition, a new LC/MS/MS-SRM method must be developed for each new candidate. To address these issues, several sample preparation kits for mass spectrometric bioanalysis of human mAbs have been developed, and sample preparation is becoming simpler [10].

The 'universal surrogate peptide approach' is another LC/MS/MS-SRM method for mAbs analysis in which a common tryptic peptide from the constant region of the mAb is used as a surrogate peptide and such a method was reported previously [11-17]. Furlong et al. demonstrated that LC/MS/MS-SRM using the surrogate peptide 'VVSVLTVLHQDWLNGK' from the heavy chain of human IgG1, 3 and 4 is suitable for the bioanalysis of diverse human $\mathrm{F}_{\mathrm{C}}$ region-containing therapeutic protein candidates in plasma samples in animal studies [12]. They also reported the 'dual universal peptide approach' using two surrogate peptides from the heavy and light chains, providing increased insight into structural integrity and increased confidence in the study data [17]. In addition to the conventional SRM assay using a triple quadrupole mass spectrometer, an SRM assay using a high-resolution mass spectrometer was also applied for quantitative bioanalysis using a universal surrogate peptide approach [11]. Previous reports suggest that one optimized universal surrogate peptide approach is applicable for the bioanalysis of several therapeutic proteins containing the human $\mathrm{F}_{\mathrm{c}}$-region in animal studies, leading to rapid evaluation of $\mathrm{mAb}$ candidates in nonclinical animal studies. However, the robustness and interlaboratory reproducibility of this widely usable approach remains unclear, which hampers its general use. Therefore, we believed that it would be valuable to verify a generic universal peptide approach in multiple laboratories.

In this study, we aimed to develop a simple, robust and low-cost generic LC/MS/MS-SRM approach for target $\mathrm{mAb}$ quantification in nonclinical animal studies. We first optimized the sample preparation for $\mathrm{mAbs}$ in spiked mouse serum samples, and then evaluated the feasibility of using an LC/MS/MS-SRM method containing the optimized sample preparation method in a collaborative study involving six laboratories, including those from pharmaceutical companies and CROs. Anti-CD20 mAb (rituximab) and anti-Her2 mAb (trastuzumab) were used as model therapeutic mAbs.

\section{Materials \& methods}

Therapeutic mAbs \& mouse sera

Commercially available rituximab and trastuzumab products were used in this study. Pooled sera from normal mice were purchased from Cedarlane Laboratories (Ontario, Canada), Kohjin Bio (Saitama, Japan), Fitzgerald Industries International (MA, USA), Rockland Immunochemicals (PA, USA), Abcam (Cambridge, UK) and Innovative Research (MI, USA).

\section{Reagents \& chemicals}

The reagents and chemicals used for sample preparation in Lab A are listed in Supplementary Table 1. Trypsin (Trypsin Gold, Promega, WI, USA), synthetic stable isotope-labeled (SIL) peptide (VVSVLTVLHQDWL $\left({ }^{13} \mathrm{C}_{6} ;{ }^{15} \mathrm{~N}\right) \mathrm{NGK}$, SCRUM, Tokyo, Japan), SIL mAb (catalog number; MSQC3, MilliporeSigma, MO, USA), and Oasis HLB $\mu$ Elution plate (Waters, MA, USA) were common materials in the collaborative study.

\section{Preparation of stock solutions, serum calibration standards \& quality control samples}

Stock solutions of rituximab were prepared using phosphate-buffered saline (PBS) at concentrations of 0.01 , $0.05,0.10,1.00,5.00$ and $10.00 \mathrm{mg} / \mathrm{ml}$. Calibration standards and quality control (QC) samples were prepared by dilution of the rituximab stock solutions with pooled mouse serum. Final concentrations of the calibration standards were adjusted to concentrations of $1.0,5.0,10.0,50.0,100.0,500.0$ and $1000.0 \mu \mathrm{g} / \mathrm{ml}$, and the QC samples for the collaborative study were prepared at a concentration of $1.0 \mu \mathrm{g} / \mathrm{ml}$. Similarly, the stock solutions, serum calibration standards and QC samples of trastuzumab were prepared, and the QC concentrations were 1.0, $3.0,50.0$ and $800.0 \mu \mathrm{g} / \mathrm{ml}$. 
Table 1. The optimized sample preparation method (internal standard: stable isotope-labeled peptide).

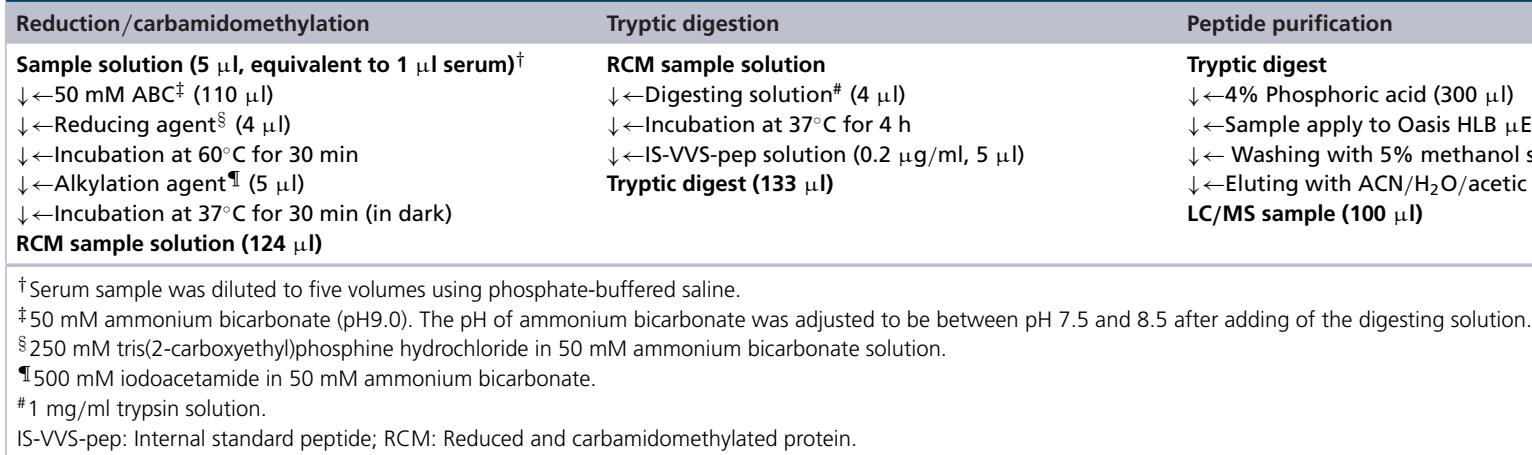

† Serum sample was diluted to five volumes using phosphate-buffered saline.

$\ddagger 50 \mathrm{mM}$ ammonium bicarbonate (pH9.0). The $\mathrm{pH}$ of ammonium bicarbonate was adjusted to be between $\mathrm{pH} 7.5$ and 8.5 after adding of the digesting solution.

$\S 250 \mathrm{mM}$ tris(2-carboxyethyl)phosphine hydrochloride in $50 \mathrm{mM}$ ammonium bicarbonate solution.

I $500 \mathrm{mM}$ iodoacetamide in $50 \mathrm{mM}$ ammonium bicarbonate.

\#1 $\mathrm{mg} / \mathrm{ml}$ trypsin solution.

IS-VVS-pep: Internal standard peptide; RCM: Reduced and carbamidomethylated protein.

\section{Internal standard solution}

The synthetic SIL peptide (VVSVLTVLHQDWL $\left[{ }^{13} \mathrm{C}_{6} ;{ }^{15} \mathrm{~N}\right] \mathrm{NGK}$ ) was used as an internal standard peptide (IS-VVS-pep). The IS-VVS-pep stock solution was prepared at $1.0 \mathrm{mg} / \mathrm{ml}$ using DMSO. An internal standard peptide solution at $0.2 \mu \mathrm{g} / \mathrm{ml}$ (IS-VVS-pep solution) was prepared by diluting the stock solution with DMSO solution.

The SIL mAb was used as an internal standard for the whole mAb (IS-whole mAb). The IS-whole mAb stock solution was prepared at a concentration of $200.0 \mu \mathrm{g} / \mathrm{ml}$ using $0.1 \%$ formic acid solution. An IS-whole mAb solution at $10.0 \mu \mathrm{g} / \mathrm{ml}$ was prepared by diluting the stock solution with a $0.1 \%$ formic acid solution.

The IS-VVS-pep solution was added to the sample mixture after tryptic digestion, and the IS-whole mAb solution was added before reduction/carbamidomethylation.

\section{Sample preparation}

Sample preparation protocols

Serum samples were diluted with four volumes of PBS, and $5 \mu$ l of a diluted sample solution (equivalent to $1 \mu$ l serum) was used as the starting serum sample for sample preparation. The optimized sample preparation methods are summarized in Table 1 and Supplementary Table 2. The methods consisted of the following three processes: carbamidomethylation, tryptic digestion and peptide purification.

\section{Optimization of the tryptic digestion time}

The pooled mouse serum spiked with $1.0 \mu \mathrm{g} / \mathrm{ml}$ rituximab was used as the rituximab sample solution $(S)$. The pooled mouse serum alone was used as the matrix sample solution (M). The $S$ and $M$ solutions were treated according to Table 1. As an additional process, after digestions for 1, 2, 4, 6 and $16 \mathrm{~h}$, synthetic nonlabeled VVS-pep (SCRUM, Tokyo, Japan) was spiked into M (now named as M1) at a molar concentration equal to that of the VVS-pep from rituximab in S (called S1). The samples were analysed with the analytical conditions of Lab A (Supplementary Table 3). The digestion efficiency (\%) was calculated by the following formula. The analyses of $\mathrm{S} 1$ and M1 were performed in triplicate.

$$
\text { Digestion efficacy }(\%)=\frac{\text { average peak area of } S 1}{\text { average peak area of } M 1} \times 100
$$

Peptide recovery of the peptide purification process

Two kinds of matrix sample solutions ( $\mathrm{S}$ and $\mathrm{M}$ ) were treated according to Table 1 . Additionally, after digestion, $S$ (now called S2) before purification and M (now called M2) after purification were spiked with synthetic nonlabeled VVS-pep at a molar concentration equal to 1.0, 10.0, 50.0, 100.0, 500.0 and $1000.0 \mu \mathrm{g} / \mathrm{ml}$ of rituximab. The sample solutions were analysed by Lab A. The peptide recovery $(\%)$ of the peptide purification process was calculated by the following formula. The analyses of S2 and M2 were performed in triplicate.

$$
\text { Peptide recovery }(\%) \text { of the purification process }=\frac{\text { average peak area of } S 2}{\text { average peak area of } M 2} \times 100
$$


Table 2. Parameter and acceptance criteria of analytical method validation used in this study.

\begin{tabular}{|c|c|}
\hline Selectivity & Six lots of blanks: $<20 \%$ of LLOQ for analyte, $<5 \%$ of IS \\
\hline Linearity of calibration curve & $\begin{array}{l}\text { At least } 75 \% \text { of the calibration standards, with a minimum of six points, including the LLOQ and the } \\
\text { highest points, meet the criteria } \\
r>0.98 \\
\text { Mean accuracy of each point: within } 20 \% \text { (LLOQ: within } \pm 25 \% \text { ) } \\
\text { Precision of each point: } \leq 20 \% \text { (LLOQ: } \leq 25 \% \text { ) }\end{array}$ \\
\hline LLOQ & $\begin{array}{l}\text { Minimum } 3 \text { runs } \\
\text { Mean accuracy: within } \pm 25 \% \\
\text { Precision: } \leq 25 \%\end{array}$ \\
\hline Accuracy and precision & $\begin{array}{l}\text { Mean accuracy of each QC sample: within } \pm 20 \% \text { (LLOQ: within } \pm 25 \% \text { ) } \\
\text { Precision of each QC sample: } \leq 20 \% \text { (LLOQ: } \leq 25 \% \text { ) }\end{array}$ \\
\hline Matrix effect & $\begin{array}{l}\text { Six lots } \\
\text { Evaluation using QC samples } \\
\text { Precision: } \leq 20 \%\end{array}$ \\
\hline Carryover & $<20 \%$ of LLOQ for analyte \\
\hline Dilution integrity & Mean accuracy: within $\pm 20 \%$, precision: $\leq 20 \%$ \\
\hline $\begin{array}{l}\text { Processed sample stability (stability of MS sample } \\
\text { in auto sampler) }\end{array}$ & Mean accuracy: within $\pm 20 \%$ \\
\hline
\end{tabular}

Collaborative study

The analytical performance of the method was evaluated in terms of selectivity, linearity, accuracy and precision by LSI Medience Corp., Sumika Chemical Analysis Service, Ltd, Sekisui Medical Co. Ltd, Daiichi Sankyo Co., Ltd, Towa Pharmaceutical Co. Ltd, and the National Institute of Health Sciences. The accuracy and precision were calculated based on the measurement results of the QC sample $(1.0 \mu \mathrm{g} / \mathrm{ml}, \mathrm{n}=3)$ by the following formulas:

$$
\begin{aligned}
& \text { Accuracy }(\%)=\frac{\text { measurmental }}{\text { theoretical }} \times 100 \\
& \text { Precision CV }(\%)=\frac{\text { standard deviation }}{\text { average }} \times 100
\end{aligned}
$$

The criteria for selectivity, linearity, precision and accuracy were set referring to previous reports (Table 2) [18].

\section{Applicability evaluation}

To evaluate the applicability of our method for other mAbs, method validation (selectivity, linearity, LLOQ, accuracy and precision, matrix effect, carryover and stability of the processed sample in the auto sampler) was performed using trastuzumab as a model mAb and IS-VVS-pep as an internal standard by Lab A. The criteria of the method validation are compiled in Table 2.

\section{Results \& discussion}

\section{Selection of the surrogate peptide}

Rituximab and trastuzumab contain the constant regions of the $\gamma 1$-chain and $\kappa$-chain of human antibodies. Supplementary Table 4 shows typical surrogate peptide candidates derived from the $\gamma 1$-chain and $\kappa$-chain in previous reports $[11,12,17]$. First, we confirmed the ion intensities of these candidates from rituximab. As shown in Figure 1A, the triply charged ion at $m / z 603.4$ of the peptide VVSVLTVLHQDWLNGK (VVS-pep) from the human $\gamma 1$-chain indicated the highest intensity. In addition, its MS/MS spectrum showed preferential generation of the $y_{14}^{2+}$ fragment ion at $m / z 805.7$ (Figure 1B). The VVS-pep underwent less intensity loss during MS/MS fragmentation, therefore, we decided to use this peptide as surrogate peptide in this study. The Q1 and Q3 transitions were set to $m / z 603-604\left(3^{+}\right)$and $m / z$ 805-806 $\left(2^{+}\right)$, respectively. The transitions of the two internal standards, IS-VVS-pep and IS-whole mAb, were set to $m / z 606$ (Q1) and $m / z 809$ (Q3), and $m / z 606$ (Q1) and $m / z$ 809-810 (Q3), respectively. Meanwhile, the $y_{11}{ }^{2+}, y_{12}{ }^{2+}$ and $y_{15}{ }^{2+}$ fragment ions were also good candidates for the Q3 ion. When any interferences are problems, using these multiple fragment ions would be more suitable for quantification. 


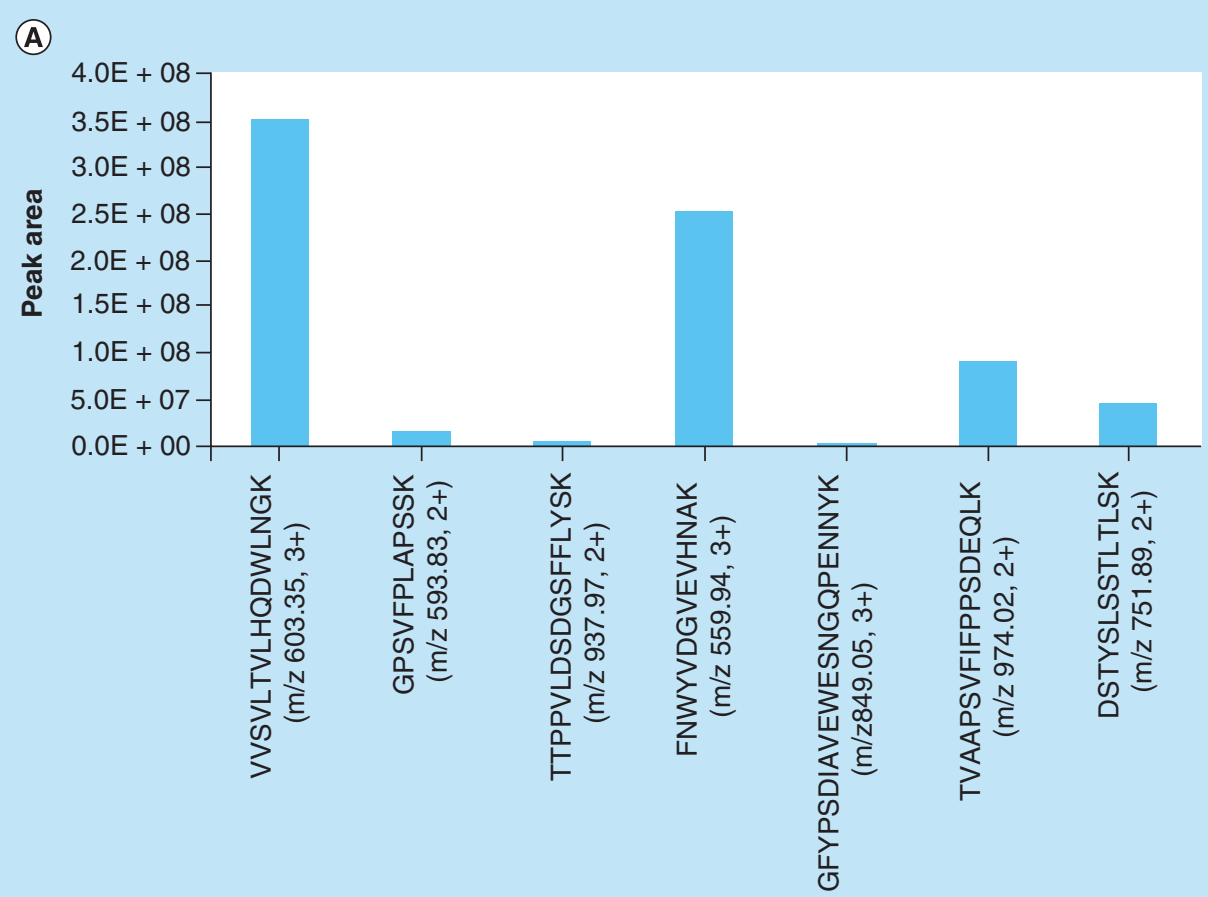

Peptide sequence
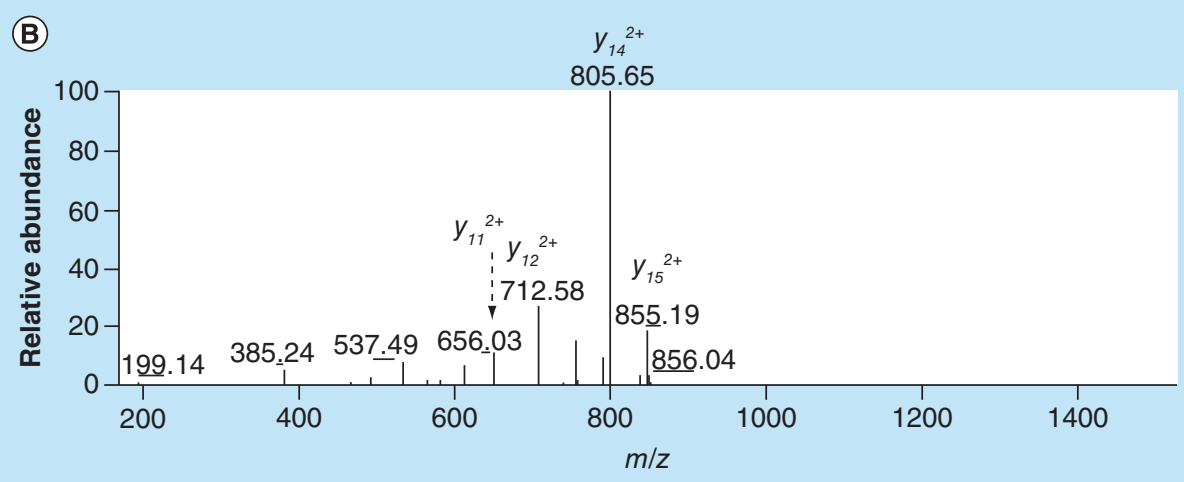

Figure 1. Selection of surrogate peptide.

(A) The ion intensities of precursor ions from the surrogate peptide candidates. (B) The fragmentation pattern of a surrogate peptide, VVSVLTVLHQDWLNGK, from human $\gamma 1$-chain obtained by LC/MS/MS.

\section{Optimization of sample preparation}

We designed the conditions of the reduction/carbamidomethylation, tryptic digestion and peptide purification processes by referring to previous reports [11-16]. We first evaluated the efficiencies (\%) of the tryptic digestion process shown in Table 1 while varying the digestion time at 1, 2, 4, 6 and $16 \mathrm{~h}$ (Figure 2A): the most effective digestion time was between 2 and $4 \mathrm{~h}$ (78-79\%). Interestingly, longer digestion times (6 and $16 \mathrm{~h})$ decreased the efficiency. Although the detailed reason for this decreased efficiency was unclear, ion suppression with an increase in tryptic peptide generations, peptide stability in the reaction solution and/or nonspecific digestion may be involved. Based on the results, the tryptic digestion time was set to $4 \mathrm{~h}$ in our method.

Next, we verified the recovery (\%) of the surrogate peptide after purification (Figure 2B). When mouse sera sample solutions containing nonlabeled VVS-pep corresponding to rituximab at 1.0, 10.0, 50.0, 100.0, 500.0 and $1000.0 \mu \mathrm{g} / \mathrm{ml}$ were tested, the peptide recoveries (\%) of all sample solutions were almost constant at 70-80\% 


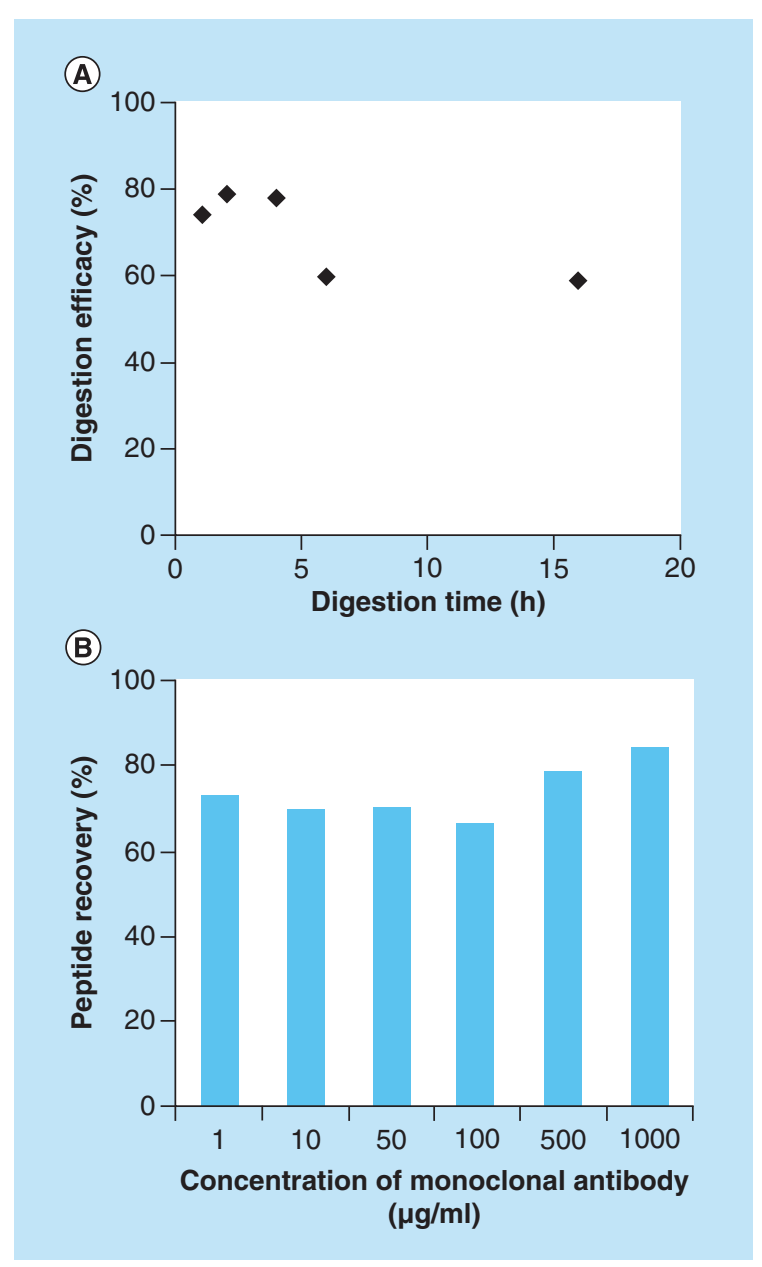

Figure 2. Optimization of sample preparation processes.

(A) The influence of tryptic digestion time on peptide recovery. (B) The influence of the concentration of monoclonal antibody in the serum sample on peptide recovery. The values in the panels were calculated using the average values of triplicate experiments $(n=3)$.

recovery. We confirmed that the performance of our peptide purification process was suitable in the range of 1.0 to $1000.0 \mu \mathrm{g} / \mathrm{ml} \mathrm{mAb}$.

\section{Optimization of LC/MS conditions}

The optimal LC/MS conditions are dependent on the specific LC/MS system, including the type of analytical column, in each laboratory. Therefore, we optimized the LC/MS conditions in each laboratory (Supplementary Table 3).

\section{Evaluation of method feasibility through a collaborative study on rituximab}

As shown in the previous sections, we successfully designed an LC/MS/MS-SRM method with sample preparation using IS-VVS-pep or IS-whole mAb (Table 1 \& Supplementary Table 2). To confirm the feasibility of the methods, its analytical performance was evaluated in terms of selectivity, linearity, accuracy and precision through a collaborative study involving six laboratories. Commercially available therapeutic rituximab was used as a model $\mathrm{mAb}$. The same lots of rituximab, normal mouse sera, trypsin and internal standards (IS-VVS-pep and IS-whole $\mathrm{mAb}$ ) were used as common materials.

\section{Selectivity}

Selectivity was evaluated using double and single blank samples obtained from six individual mouse serum samples. Single blank samples were prepared by spiking appropriate amounts of the internal standards (IS-VVS-pep or IS-whole $\mathrm{mAb}$ ) into each mouse serum sample in accordance with Table 1 and Supplementary Table 2. As shown in Table 3, the interfering peaks $>20 \%$ of the LLOQ $(1.0 \mu \mathrm{g} / \mathrm{ml})$ responses of the analyte (VVS-pep), and $>5 \%$ of the internal standard responses were not observed in any double blank or single blank samples from any of the laboratories, indicating acceptable selectivity of our method. 
Table 3. Selectivity evaluation using six individual mouse sera.

\begin{tabular}{|c|c|c|c|c|c|c|c|c|c|c|c|c|c|}
\hline \multirow[t]{2}{*}{ Lab } & \multirow[t]{2}{*}{ Double or single blank } & \multicolumn{6}{|c|}{$\begin{array}{c}\text { Interference peak area against analyte LLOQ peak } \\
\text { area (\%) }\end{array}$} & \multicolumn{6}{|c|}{ Interference peak area against IS peak area (\%) } \\
\hline & & M1 & M2 & M3 & M4 & M5 & M6 & M1 & M2 & M3 & M4 & M5 & M6 \\
\hline \multirow[t]{3}{*}{ A } & Double & 0.0 & 3.7 & 0.0 & 0.0 & 0.0 & 0.0 & $0.1 / 0.0^{\dagger}$ & $0.1 / 0.0$ & $0.2 / 0.0$ & $0.2 / 0.0$ & $0.2 / 0.0$ & $0.2 / 0.0$ \\
\hline & Single (IS-VVS-pep) & 2.4 & 1.0 & 0.0 & 0.0 & 2.8 & 0.0 & $N / A^{\ddagger}$ & $N / A$ & $\mathrm{~N} / \mathrm{A}$ & $\mathrm{N} / \mathrm{A}$ & $\mathrm{N} / \mathrm{A}$ & $\mathrm{N} / \mathrm{A}$ \\
\hline & Single (IS-whole mAb) & 3.0 & 9.1 & 4.9 & 9.0 & 0.0 & 5.7 & $\mathrm{~N} / \mathrm{A}$ & $\mathrm{N} / \mathrm{A}$ & $\mathrm{N} / \mathrm{A}$ & N/A & $\mathrm{N} / \mathrm{A}$ & $\mathrm{N} / \mathrm{A}$ \\
\hline \multirow[t]{3}{*}{ B } & Double & 0.0 & 0.0 & 0.0 & 0.0 & 0.0 & 0.0 & $0.0 / 0.0$ & $0.0 / 0.0$ & $0.0 / 0.0$ & $0.0 / 0.0$ & $0.0 / 0.0$ & $0.0 / 0.0$ \\
\hline & Single (IS-VVS-pep) & 0.0 & 0.0 & 8.3 & 0.0 & 0.0 & 6.2 & $\mathrm{~N} / \mathrm{A}$ & $\mathrm{N} / \mathrm{A}$ & $\mathrm{N} / \mathrm{A}$ & $\mathrm{N} / \mathrm{A}$ & $\mathrm{N} / \mathrm{A}$ & $\mathrm{N} / \mathrm{A}$ \\
\hline & Single (IS-whole mAb) & 0.0 & 8.5 & 0.0 & 0.0 & 0.0 & 0.0 & $\mathrm{~N} / \mathrm{A}$ & $\mathrm{N} / \mathrm{A}$ & $\mathrm{N} / \mathrm{A}$ & $\mathrm{N} / \mathrm{A}$ & $\mathrm{N} / \mathrm{A}$ & $\mathrm{N} / \mathrm{A}$ \\
\hline \multirow[t]{3}{*}{ C } & Double & 0.0 & 8.7 & 0.0 & 5.5 & 0.0 & 0.0 & $0.0 / 0.0$ & $0.0 / 0.0$ & $0.0 / 0.0$ & $0.0 / 0.0$ & $0.0 / 0.0$ & $0.0 / 0.0$ \\
\hline & Single (IS-VVS-pep) & 0.0 & 0.0 & 0.0 & 0.0 & 0.0 & 5.6 & $\mathrm{~N} / \mathrm{A}$ & $\mathrm{N} / \mathrm{A}$ & $\mathrm{N} / \mathrm{A}$ & N/A & $\mathrm{N} / \mathrm{A}$ & $\mathrm{N} / \mathrm{A}$ \\
\hline & Single (IS-whole mAb) & 7.3 & 2.9 & 0.0 & 0.0 & 0.0 & 2.3 & $\mathrm{~N} / \mathrm{A}$ & $\mathrm{N} / \mathrm{A}$ & $\mathrm{N} / \mathrm{A}$ & $\mathrm{N} / \mathrm{A}$ & $\mathrm{N} / \mathrm{A}$ & $\mathrm{N} / \mathrm{A}$ \\
\hline \multirow[t]{3}{*}{ D } & Double & 0.0 & 0.0 & 0.0 & 8.0 & 7.4 & 8.4 & $0.0 / 0.0$ & $0.0 / 0.0$ & $0.0 / 0.0$ & $0.0 / 0.0$ & $0.0 / 0.0$ & $0.0 / 0.0$ \\
\hline & Single (IS-VVS-pep) & 0.0 & 0.0 & 13.8 & 10.5 & 0.0 & 0.0 & $\mathrm{~N} / \mathrm{A}$ & $\mathrm{N} / \mathrm{A}$ & $\mathrm{N} / \mathrm{A}$ & N/A & $\mathrm{N} / \mathrm{A}$ & $\mathrm{N} / \mathrm{A}$ \\
\hline & Single (IS-whole mAb) & 0.0 & 0.0 & 0.0 & 0.0 & 0.0 & 0.0 & $\mathrm{~N} / \mathrm{A}$ & $\mathrm{N} / \mathrm{A}$ & $\mathrm{N} / \mathrm{A}$ & N/A & $\mathrm{N} / \mathrm{A}$ & $\mathrm{N} / \mathrm{A}$ \\
\hline \multirow[t]{3}{*}{$E$} & Double & 0.0 & 0.0 & 0.0 & 0.0 & 0.0 & 0.0 & $0.0 / 0.0$ & $0.0 / 0.0$ & $0.0 / 0.0$ & $0.0 / 0.0$ & $0.0 / 0.0$ & $0.0 / 0.0$ \\
\hline & Single (IS-VVS-pep) & 0.0 & 0.0 & 0.0 & 0.0 & 0.0 & 0.0 & $\mathrm{~N} / \mathrm{A}$ & $\mathrm{N} / \mathrm{A}$ & $\mathrm{N} / \mathrm{A}$ & N/A & $\mathrm{N} / \mathrm{A}$ & $\mathrm{N} / \mathrm{A}$ \\
\hline & Single (IS-whole mAb) & 0.0 & 0.0 & 0.0 & 0.0 & 0.0 & 0.0 & $\mathrm{~N} / \mathrm{A}$ & $\mathrm{N} / \mathrm{A}$ & $\mathrm{N} / \mathrm{A}$ & $\mathrm{N} / \mathrm{A}$ & $\mathrm{N} / \mathrm{A}$ & $\mathrm{N} / \mathrm{A}$ \\
\hline \multirow[t]{3}{*}{$\mathrm{F}$} & Double & 0.0 & 0.0 & 0.0 & 0.0 & 0.0 & 0.0 & $0.0 / 0.0$ & $0.0 / 0.0$ & $0.0 / 0.0$ & $0.0 / 0.0$ & $0.0 / 0.0$ & $0.0 / 0.0$ \\
\hline & Single (IS-VVS-pep) & 0.0 & 0.0 & 0.0 & 0.0 & 0.0 & 0.0 & $\mathrm{~N} / \mathrm{A}$ & $\mathrm{N} / \mathrm{A}$ & $\mathrm{N} / \mathrm{A}$ & $\mathrm{N} / \mathrm{A}$ & $\mathrm{N} / \mathrm{A}$ & $\mathrm{N} / \mathrm{A}$ \\
\hline & Single (IS-whole mAb) & 0.0 & 0.0 & 0.0 & 0.0 & 0.0 & 0.0 & $\mathrm{~N} / \mathrm{A}$ & $\mathrm{N} / \mathrm{A}$ & $\mathrm{N} / \mathrm{A}$ & $\mathrm{N} / \mathrm{A}$ & $\mathrm{N} / \mathrm{A}$ & $\mathrm{N} / \mathrm{A}$ \\
\hline$¥$ Not & $\begin{array}{l}\text { indicate the interferenc } \\
\text { able. }\end{array}$ & die & $-v$ & an & nol & & ep/l & hole $\mathrm{mA}$ & & & ory. & & \\
\hline
\end{tabular}

\section{Linearity of the calibration curve}

The linearity of the calibration curve of our method was evaluated using a linear regression model after measuring seven calibration standards spiked with rituximab $(1.0,5.0,10.0,50.0,100.0,500.0$ and $1000.0 \mu \mathrm{g} / \mathrm{ml} ; \mathrm{n}=1)$. The weighted calibration plot was generated using the $1 / \mathrm{x}^{2}$ method in all the laboratories. The calibration curve obtained by Lab A is shown in Figure 3. The calibration fit formulas were $Y=0.030 X-0.0066$ (correlation coefficient $[r]=0.9988)$ for IS-VVS-pep and $Y=0.037 X-0.0070(r=0.9989)$ for IS-whole mAb. The accuracy obtained for the calibration standards ranged from 90.7 to $105.5 \%$. Table 4 shows the parameters of the calibration curves from all the laboratories. The calibration curves obtained using IS-VVS-pep and IS-whole mAb showed good linearity from 1.0 to $1000.0 \mu \mathrm{g} / \mathrm{ml}$ ( $r$ values from 0.9939 to 0.9992$)$, with good accuracy (83.0-114.7\%) for the calibration standards.

\section{Accuracy \& precision}

Accuracy and precision were determined by analysis of the $1.0 \mu \mathrm{g} / \mathrm{ml} \mathrm{QC}$ sample. The measurement was performed in triplicate. When IS-VVS-pep was used as an internal standard, the mean accuracy and precision were 102.3$114.3 \%$ and $3.5-11.4 \%$, respectively, and when IS-whole was used as an internal standard, these parameters were 82.4-111.0\% and 2.5-8.7\%, respectively (Table 5). When IS-VVS-pep was used as internal standard, the accuracy was above $100 \%$, suggesting the low recovery of the standard peptide. However, the same trend was not observed in QC assays using trastuzumab (Supplementary Table 7). These results suggested that the increased trend was not specific to the standard peptide. In addition, the difference between internal standard peptide (IS-pep) and IS-whole $\mathrm{mAb}$ did not influence the variability of our SRM assay. In the case of SRM assay without affinity purification process, IS-pep might be enough as internal standard.

Collectively, the feasibility of using LC/MS/MS-SRM with our sample preparation method was demonstrated in all laboratories regardless of the different analytical conditions of the instrumentation and LC/MS systems in each laboratory. Therefore, our method is sufficiently robust. 
(A)

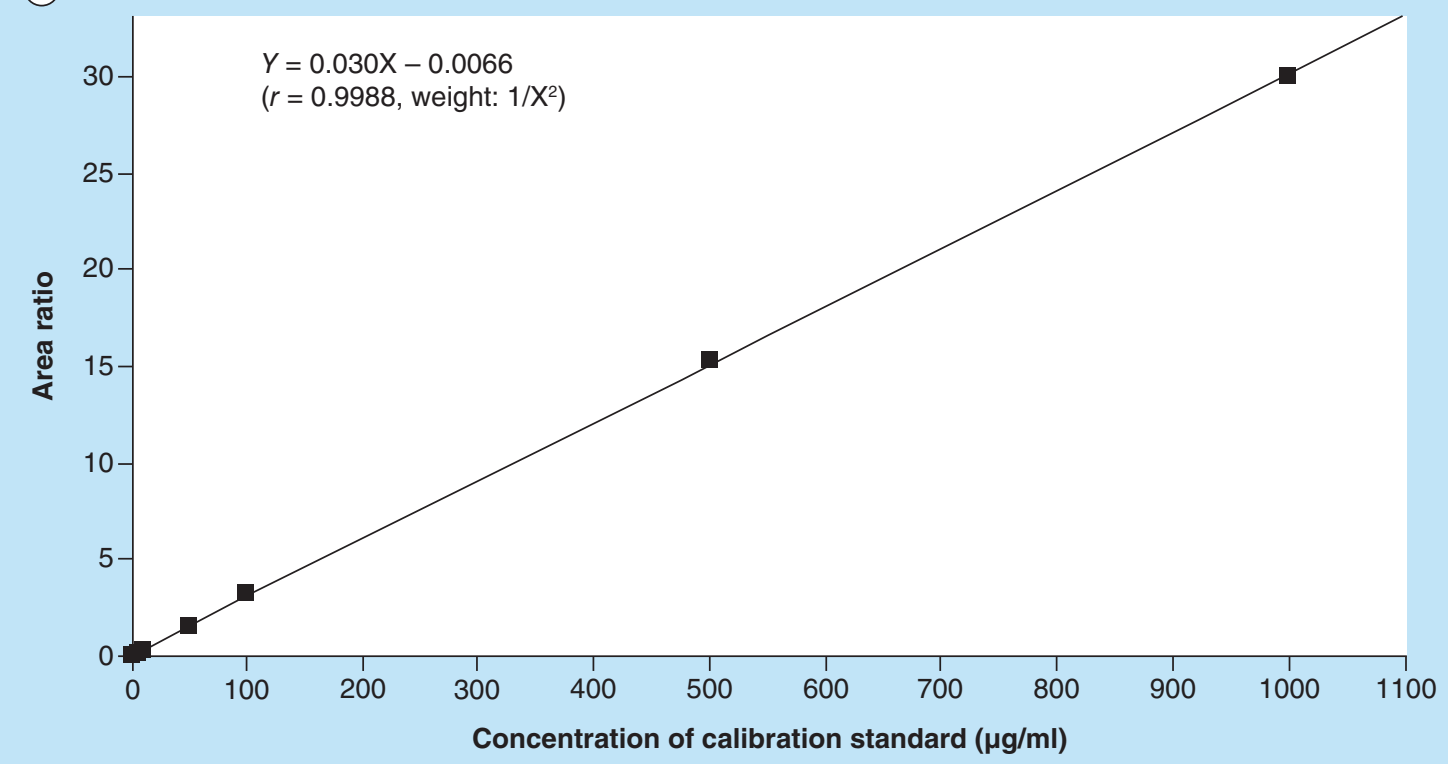

(B)

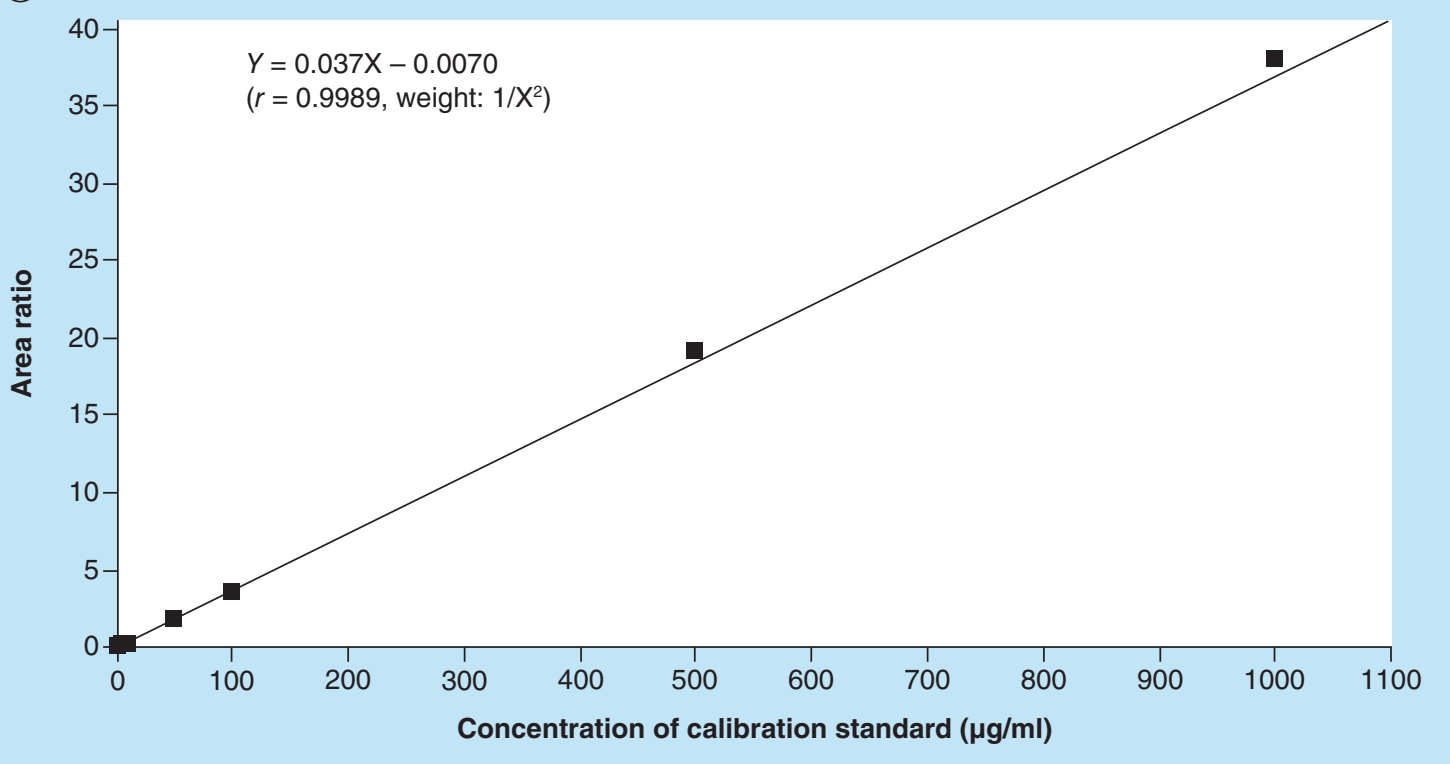

Figure 3. Calibration curves for the quantification of rituximab in mouse serum obtained by laboratory $A$.

(A) The calibration curve using IS-VVS-pep (internal standard peptide) and (B) the calibration curve using internal standard-whole monoclonal antibody.

\section{Applicability evaluation with trastuzumab}

The applicability of our method was evaluated by method validation using calibration standards and QC samples spiked with another $\mathrm{mAb}$, trastuzumab. The evaluation was performed by only Lab. A and the results are summarized in Supplementary Tables 5-11.

\section{Selectivity}

Selectivity was evaluated using double blank samples obtained from six individual mouse serum samples. Interfering peaks for the analyte (VVS-pep) and internal standard (IS-VVS-pep) were detected in the SRM chromatograms 


\begin{tabular}{|c|c|c|c|c|c|c|c|c|c|c|c|}
\hline \multirow[t]{2}{*}{ Lab } & \multirow[t]{2}{*}{ IS } & \multirow[t]{2}{*}{ Slope } & \multirow[t]{2}{*}{ Intercept } & \multirow[t]{2}{*}{$r$} & \multicolumn{7}{|c|}{ Nominal concentration of monoclonal antibody $(\mu \mathrm{g} / \mathrm{ml})$, accuracy $(\%)$} \\
\hline & & & & & 1000.0 & 500.0 & 100.0 & 50.0 & 10.0 & 5.0 & 1.0 \\
\hline A & $\begin{array}{l}\text { IS-whole } \\
\text { mAb }\end{array}$ & 0.037 & -0.0070 & 0.9989 & 103.3 & 103.6 & 97.6 & 104.4 & 93.1 & 96.7 & 101.3 \\
\hline B & IS-VVS-pep & 0.075 & -0.0174 & 0.9980 & 95.5 & 103.4 & 111.0 & 98.4 & 95.8 & 94.0 & 102.0 \\
\hline \multirow[t]{2}{*}{ c } & IS-VVS-pep & 0.089 & -0.0031 & 0.9992 & 94.9 & 105.6 & 96.4 & 102.2 & 99.1 & 102.2 & 99.6 \\
\hline & $\begin{array}{l}\text { IS-whole } \\
\text { mAb }\end{array}$ & 0.146 & 0.0110 & 0.9988 & 95.7 & 103.8 & 101.0 & 94.4 & 107.0 & 97.6 & 99.8 \\
\hline \multirow[t]{2}{*}{ D } & IS-VVS-pep & 0.021 & 0.0010 & 0.9939 & 112.0 & 108.4 & 107.0 & 92.2 & 93.8 & 83.0 & 104.0 \\
\hline & $\begin{array}{l}\text { IS-whole } \\
\text { mAb }\end{array}$ & 0.074 & -0.0213 & 0.9983 & 98.9 & 101.2 & 104.0 & 105.0 & 101.0 & 88.2 & 102.0 \\
\hline \multirow[t]{2}{*}{$\mathrm{F}$} & IS-VVS-pep & 0.303 & -0.0750 & 0.9981 & 92.3 & 98.4 & 103.4 & 108.0 & 103.9 & 93.1 & 100.8 \\
\hline & $\begin{array}{l}\text { IS-whole } \\
\text { mAb }\end{array}$ & 0.272 & -0.0780 & 0.9952 & 87.9 & 91.2 & 104.6 & 98.0 & 114.7 & 106.3 & 97.3 \\
\hline
\end{tabular}

IS-VVS-pep: Internal standard peptide; IS-whole mAb: Internal standard for the whole monoclonal antibody; Lab: Laboratory; r: Correlation coefficient.

Table 5. Accuracy and precision $(1.0 \mu \mathrm{g} / \mathrm{ml}, \mathrm{n}=3)$.

\begin{tabular}{|c|c|c|c|c|}
\hline \multirow[t]{3}{*}{ Laboratory } & \multicolumn{4}{|c|}{ Internal standard } \\
\hline & \multicolumn{2}{|c|}{ IS-VVS-pep } & \multicolumn{2}{|c|}{ IS-whole mAb } \\
\hline & Mean accuracy (\%) & Precision (CV\%) & Mean accuracy (\%) & Precision (CV\%) \\
\hline$A$ & 105.9 & 5.5 & 91.8 & 6.8 \\
\hline B & 112.1 & 11.0 & 111.0 & 6.8 \\
\hline C & 107.3 & 3.5 & 110.7 & 8.7 \\
\hline D & 114.3 & 8.7 & 98.5 & 8.4 \\
\hline $\mathrm{E}$ & 102.3 & 5.5 & 82.4 & 4.7 \\
\hline $\mathrm{F}$ & 102.9 & 11.4 & 92.6 & 2.5 \\
\hline
\end{tabular}

IS-VVS-pep: Internal standard peptide; IS-whole mAb: Internal standard for the whole monoclonal antibody.

of M1, M4 and M6, and of M4 and M5, respectively (Supplementary Table 5). However, interfering peaks with responses $>20 \%$ of the response of the LLOQ $(1.0 \mu \mathrm{g} / \mathrm{ml})$ of VVS-pep, and $>5 \%$ of that of IS-VVS-pep were not observed in any samples. These data confirm the acceptable selectivity of our method.

\section{Linearity of the calibration curve}

The linearity of the calibration curve was evaluated using a linear regression model after measuring seven calibration standards spiked with trastuzumab (1.0, 5.0, 10.0, 50.0, 100.0, 500.0 and $1000.0 \mu \mathrm{g} / \mathrm{ml} ; \mathrm{n}=2$ ) (Supplementary Table 6 \& Supplementary Figure 1). The weighted calibration plot was generated using the $1 / \mathrm{x}^{2} \operatorname{method}$ as the plot for rituximab was. The calibration fit formula was $Y=0.025 X-0.0069(r=0.9977)$. The accuracy of the calibration standards ranged from 85.2 to $109.4 \%$, satisfying the predefined acceptance criteria.

\section{LLOQ}

The LLOQ was defined as the lowest concentration of the calibration curve with acceptable accuracy, which was determined to be $1.0 \mu \mathrm{g} / \mathrm{ml}$ (Supplementary Table 6). The mean accuracy at the LLOQ was within $\pm 11 \%$ of the theoretical concentration, and the precision at the LLOQ was not >11\% (Supplementary Table 7). 


\section{Accuracy \& precision}

The accuracy and precision were evaluated by analysing three replicates of pooled mouse serum samples spiked with trastuzumab at four concentrations: the LLOQ, low and intermediate concentrations, and the ULOQ (i.e., 1.0, 3.0, 50.0 and $800.0 \mu \mathrm{g} / \mathrm{ml}$, respectively). As shown in Supplementary Table 7, the mean accuracy and precision at the four level concentrations were $90.6-107.7 \%$ and $2.1-10.9 \%$, respectively.

\section{Matrix effect}

Six mouse serum samples were analysed to determine the matrix effect at the low $(3.0 \mu \mathrm{g} / \mathrm{ml})$ and ULOQ $(800.0 \mu \mathrm{g} / \mathrm{ml})$ concentrations. The mean concentrations determined at the low and ULOQ were 2.7 and $825.3 \mu \mathrm{g} / \mathrm{ml}$, respectively, and the precision was within the accepted criteria $(\leq 20 \%)$ (Supplementary Table 8).

\section{Carryover}

Carryover, which is defined as residual analyte in the autosampler and column, was investigated by analyzing a double blank sample for the analyte (VVS-pep) and internal standard (IS-VVS-pep) after analyzing the highestconcentration calibration standard $(1000.0 \mu \mathrm{g} / \mathrm{ml})$. Carryover was calculated as the percent response in the double blank sample relative to that in the LLOQ sample. No carryover peaks were observed for VVS-pep and IS-VVS-pep (Supplementary Table 9).

\section{Dilution integrity}

The effect of the dilution steps in our bioanalytical method on the rituximab concentration was evaluated by preparing a mouse serum sample spiked with $1500.0 \mu \mathrm{g} / \mathrm{ml}$ rituximab, and analyzing five tenfold dilutions of this sample. The mean accuracy and precision of the diluted samples were 105.2 and $4.4 \%$, respectively. These values met the performance criteria, and therefore, suggested no analyte interference from dilution (Supplementary Table 10).

\section{Processed sample stability}

The processed sample stability at the low $(3 \mu \mathrm{g} / \mathrm{ml})$ and ULOQ $(800.0 \mu \mathrm{g} / \mathrm{ml})$ concentrations was evaluated at $5^{\circ} \mathrm{C}$ for $48 \mathrm{~h}$. The mean accuracy (\%) of the low concentration and ULOQ was 90.5 and $108.3 \%$, respectively (Supplementary Table 11).

Our group believes that the universal surrogate peptide approach is an important and useful bioanalytical method for $\mathrm{mAb}$ analysis in nonclinical animal studies due to its high versatility and applicability. To increase the generalization of this noteworthy approach, we developed a universal surrogate peptide approach by combining the original sample preparation method and a conventional SRM assay and demonstrated that this method is a useful general and robust approach for mAb analysis in a collaborative study. The most common method used in pharmacokinetic studies of rituximab and trastuzumab is ELISA. In a nonclinical pharmacokinetic study, the mean values of maximum concentration $\left(C_{\max }\right)$ in a single- and multiple-dose study of $20 \mathrm{mg} / \mathrm{kg}$ rituximab were $726.0 \pm 138.0$ and $1230 \pm 313.0 \mu \mathrm{g} / \mathrm{ml}$, respectively [19]. Additionally, it was reported that the mean value of minimum concentration $\left(C_{m i n}\right)$ in a multiple-dose study of $10 \mathrm{mg} / \mathrm{kg}$ rituximab was $59.5 \pm 64.9 \mu \mathrm{g} / \mathrm{ml}$ [20]. In another nonclinical pharmacokinetic study, the mean values of $C_{\max }$ in a single-dose study of $10 \mathrm{mg} / \mathrm{kg}$ trastuzumab were $269 \pm 52.7$ and $281 \pm 49.9 \mu \mathrm{g} / \mathrm{ml}$ [21]. Our method showed a broad dynamic range from 1.0 to $1000.0 \mu \mathrm{g} / \mathrm{ml}$, and the accuracy and precision of all calibration standards and QC samples met the predefined performance criteria. Therefore, our method can be applied to serum concentration assays of therapeutic IgG1 (and IgG4) mAbs in nonclinical studies.

Our group also discussed the acceptance criteria of accuracy and precision for method validation, based on the recommendations in previous reports $[18,22,23]$. The variability in the SRM assay results for large molecules is potentially higher than that for small molecules because the complexity of the sample preparation process is greater for the former than for the latter. Moreover, the bioanalysis of rituximab and trastuzumab have been generally performed with the LBA method; for these reasons, the criteria ( $\leq 20 \%$ [LLOQ: $\leq 25 \%$ ]) in the guideline on bioanalysis by the LBA method were adopted as the criteria of our method validation [24]. However, in our collaborative study, four laboratories achieved accuracy and precision values below the acceptance criteria (accuracy, $100 \pm 15 \%$ [LLOQ: $\pm 20 \%$ ]; precision, $\leq 15 \%$ [LLOQ: $\leq 20 \%$ ]) for the bioanalysis of small molecules using a chromatographic method [25]. Discussion on the acceptance criteria for MS-based bioanalysis of large 
molecules is still ongoing in the bioanalytical community, and the criteria have not been clearly established in any guideline/guidance. Our current data could accelerate such discussions for mAbs.

\section{Conclusion}

In this study, we developed a simple, robust and low-cost general LC/MS/MS-SRM bioanalytical method for therapeutic IgG1 mAb candidates in mouse serum by combining a developed sample preparation method and a conventional LC/MS/MS-SRM. We also demonstrated the feasibility of the method through a collaborative study involving six laboratories. This method has a sufficient calibration range $(1.0-1000.0 \mu \mathrm{g} / \mathrm{ml})$ and LLOQ $(1.0 \mu \mathrm{g} / \mathrm{ml})$ for use in animal studies and will be a useful bioanalytical method for the quantification of therapeutic IgG mAbs in nonclinical animal studies.

\section{Future perspective}

The universal surrogate peptide approach using LC/MS/MS-SRM is useful for the quantification of therapeutic $\mathrm{mAb}$ candidates in nonclinical animal studies. However, the robustness and interlaboratory reproducibility of the approach remain unclear. In this study, we developed a generic MS method for the quantification of therapeutic $\mathrm{mAb}$ candidates in mouse serum. In the future, we hope that our method will be widely used as a template method for MS-based bioanalysis of $\mathrm{mAb}$ candidates in nonclinical animal studies.

\section{Summary points}

\section{Background}

- To increase the generalization of a universal surrogate peptide approach, a generic bioanalytical method for quantification of therapeutic monoclonal antibodies (mAbs) in mouse sera was developed.

\section{Experimental}

- Rituximab and trastuzumab were used as model mAbs.

- The analytical performance of the LC/MS/MS-SRM method with the optimized sample preparation method was evaluated through a collaborative study involving six laboratories.

\section{Results \& discussion}

- A simple and easy sample preparation method consisting of carbamidomethylation, tryptic digestion and peptide purification processes was established.

- The LC/MS/MS-SRM method showed a broad dynamic range from 1.0 to $1000.0 \mu \mathrm{g} / \mathrm{ml}$.

- The selectivity, linearity of calibration curve, accuracy and precision determined by all laboratories in the collaborative study met the predefined acceptance criteria.

Conclusion

- Our method is a useful bioanalytical method for the quantification of therapeutic IgG mAbs in nonclinical animal studies.

\footnotetext{
Supplementary data

To view the supplementary data that accompany this paper please visit the journal website at: www.futurescience.com/doi/suppl/10.4155/bio-2019-0253
}

\section{Author contributions}

N Hashii designed the study and wrote the initial draft of the manuscript. N Hashii and $Y$ Tousaka contributed to the optimization of sample preparation method, and N Hashii and Y Tousaka, K Arai, R Goda, N Inoue, K Murata, T Okuzono, S Sasahara, T Shigeyama, $\mathrm{H}$ Tachiki and S Yamane contributed to the verification and validation of our LC/MS/MS-SRM method. A Ishii-Watabe and Y Saito supervised the study. All authors agree to be accountable for all aspects of the work in ensuring that questions related to the accuracy or integrity of any part of the work are appropriately investigated and resolved. The manuscript was written through contributions of all authors. All authors have given approval to the final version of the manuscript for submission.

\section{Acknowledgments}

The authors thank Y Hiruta from National Institute of Health Sciences for preparing sample solutions.

Financial \& competing interests disclosure

This work was supported by AMED under grant number JP19ak0101073. The authors have no other relevant affiliations or financial involvement with any organization or entity with a financial interest in or financial conflict with the subject matter or materials 
discussed in the manuscript apart from those disclosed. The authors have no other relevant affiliations or financial involvement with any organization or entity with a financial interest in or financial conflict with the subject matter or materials discussed in the manuscript apart from those disclosed.

No writing assistance was utilized in the production of this manuscript.

\section{Open access}

This work is licensed under the Attribution-NonCommercial-NoDerivatives 4.0 Unported License. To view a copy of this license, visit http://creativecommons.org/licenses/by-nc-nd/4.0/

\section{References}

Papers of special note have been highlighted as: $\bullet \bullet$ of considerable interest

1. Kaplon H, Reichert JM. Antibodies to watch in 2018. MAbs 10(2), 183-203 (2018).

2. Sakanaka C. Antibody therapeutics: bench to bedside. Yakugaku Zasshi 137(7), 817-822 (2017).

3. Grilo AL, Mantalaris A. The increasingly human and profitable monoclonal antibody market. Trends Biotechnol. 37(1), 9-16 (2019).

4. Fraser S, Shih JY, Ware M et al. Current trends in ligand binding real-time measurement technologies. AAPS J. 19(3), 682-691 (2017).

5. Thway TM. Fundamentals of large-molecule protein therapeutic bioanalysis using ligand-binding assays. Bioanalysis 8(1), 11-17 (2016).

6. Hoofnagle AN, Wener MH. The fundamental flaws of immunoassays and potential solutions using tandem mass spectrometry. $J$. Immunol. Methods 347(1-2), 3-11 (2009).

7. Todoroki K, Yamada T, Mizuno H, Toyo'oka T. Current mass spectrometric tools for the bioanalyses of therapeutic monoclonal antibodies and antibody-drug conjugates. Anal. Sci. 34(4), 397-406 (2018).

8. Duggan J, Ren B, Mao Y, Chen LZ, Philip E. LC-MS quantification of protein drugs: validating protein LC-MS methods with predigestion immunocapture. Bioanalysis 8(18), 1951-1964 (2016).

9. Fung EN, Bryan P, Kozhich A. Techniques for quantitative LC-MS/MS analysis of protein therapeutics: advances in enzyme digestion and immunocapture. Bioanalysis 8(8), 847-856 (2016).

10. Iwamoto N, Takanashi M, Hamada A, Shimada T. Validated LC/MS bioanalysis of rituximab CDR peptides using nano-surface and molecular-orientation limited (nSMOL) proteolysis. Biol. Pharm. Bull. 39(7), 1187-1194 (2016).

-. The innovative sample preparation method, which enables sensitive quantifications of the therapeutic monoclonal antibodies, was introduced.

11. Lanshoeft $\mathrm{C}$, Wolf $\mathrm{T}$, Heudi $\mathrm{O}$ et al. The use of generic surrogate peptides for the quantitative analysis of human immunoglobulin G1 in pre-clinical species with high-resolution mass spectrometry. Anal. Bioanal. Chem. 408(6), 1687-1699 (2016).

12. Furlong MT, Ouyang Z, Wu S et al. A universal surrogate peptide to enable LC-MS/MS bioanalysis of a diversity of human monoclonal antibody and human Fc-fusion protein drug candidates in pre-clinical animal studies. Biomed. Chromatogr. 26(8), 1024-1032 (2012).

-. It might be a first report of universal surrogate peptide approach.

13. Lee JW. Generic method approaches for monoclonal antibody therapeutics analysis using both ligand binding and LC-MS/MS techniques. Bioanalysis 8(1), 19-27 (2016).

14. Gong C, Zheng N, Zeng J, Aubry AF, Arnold ME. Post-pellet-digestion precipitation and solid phase extraction: a practical and efficient workflow to extract surrogate peptides for ultra-high performance liquid chromatography-tandem mass spectrometry bioanalysis of a therapeutic antibody in the low $\mathrm{ng} / \mathrm{ml}$ range. J. Chromatogr. A 1424, 27-36 (2015).

15. Li H, Ortiz R, Tran L et al. General LC-MS/MS method approach to quantify therapeutic monoclonal antibodies using a common whole antibody internal standard with application to preclinical studies. Anal. Chem. 84(3), 1267-1273 (2012).

16. Law WS, Genin JC, Miess C et al. Use of generic LC-MS/MS assays to characterize atypical PK profile of a biotherapeutic monoclonal antibody. Bioanalysis 6(23), 3225-3235 (2014).

17. Furlong MT, Zhao S, Mylott W et al. Dual universal peptide approach to bioanalysis of human monoclonal antibody protein drug candidates in animal studies. Bioanalysis 5(11), 1363-1376 (2013).

18. Jenkins R, Duggan JX, Aubry AF et al. Recommendations for validation of LC-MS/MS bioanalytical methods for protein biotherapeutics. AAPS J. 17(1), 1-16 (2015).

-• The validation acceptance criteria recommended in this paper are helpful for mass spectrometric bioanalysis of monoclonal antibodies.

19. Ryan AM, Sokolowski SA, Ng CK et al. Comparative nonclinical assessments of the proposed biosimilar PF-05280586 and rituximab (MabThera ${ }^{\circledR}$ ). Toxicol. Pathol. 42(7), 1069-1081 (2014).

20. Mao CP, Brovarney MR, Dabbagh K, Birnböck HF, Richter WF, Del Nagro CJ. Subcutaneous versus intravenous administration of rituximab: pharmacokinetics, CD20 target coverage and B-Cell depletion in cynomolgus monkeys. PLoS ONE 8(11), e80533 (2013). 
21. Hurst S, Ryan AM, Ng CK et al. Comparative nonclinical assessments of the proposed biosimilar PF-05280014 and trastuzumab (Herceptin $\left.{ }^{\circledR}\right)$. BioDrugs 28(5), 451-459 (2014).

22. Knutsson M, Schmidt R, Timmerman P. LC-MS/MS of large molecules in a regulated bioanalytical environment - which acceptance criteria to apply? Bioanalysis 5(18), 2211-2214 (2013).

23. Booth BP, Furmanski B. Hybrid assays: the next big thing? Bioanalysis 10(13), 975-977 (2018).

24. Claeys KC, Hopkins TL, Vega AD, Heil EL. Fluoroquinolone restriction as an effective antimicrobial stewardship intervention. Curr. Infect. Dis. Rep. 20(5), 7 (2018).

25. Lauritsen CG, Chua AL, Nahas SJ. Current treatment options: headache related to menopause-diagnosis and management. Curr. Treat. Options Neurol. 20(4), 7 (2018). 